\title{
Optimization and Calibration of Slat Position for a SPECT With Slit-Slat Collimator and Pixelated Detector Crystals
}

\author{
Xiao Deng, Tianyu Ma, Member, IEEE, Roger Lecomte, Member, IEEE, and Rutao Yao, Member, IEEE
}

\begin{abstract}
To expand the availability of SPECT for biomedical research, we developed a SPECT imaging system on an existing animal PET detector by adding a slit-slat collimator. As the detector crystals are pixelated, the relative slat-to-crystal position (SCP) in the axial direction affects the photon flux distribution onto the crystals. The accurate knowledge of SCP is important to the axial resolution and sensitivity of the system. This work presents a method for optimizing SCP in system design and for determining SCP in system geometrical calibration. The optimization was achieved by finding the SCP that provides higher spatial resolution in terms of average-root-mean-square $(\overline{R M S})$ width of the axial point spread function (PSF) without loss of sensitivity. The calibration was based on the least-square-error method that minimizes the difference between the measured and modeled axial point spread projections. The uniqueness and accuracy of the calibration results were validated through a singular value decomposition (SVD) based approach. Both the optimization and calibration techniques were evaluated with Monte Carlo (MC) simulated data. We showed that the $\overline{R M S}$ was improved about $15 \%$ with the optimal SCP as compared to the least-optimal SCP, and system sensitivity was not affected by SCP. The SCP error achieved by the proposed calibration method was less than 0.04 $\mathrm{mm}$. The calibrated SCP value was used in MC simulation to generate the system matrix which was used for image reconstruction. The images of simulated phantoms showed the expected resolution performance and were artifact free. We conclude that the proposed optimization and calibration method is effective for the slit-slat collimator based SPECT systems.
\end{abstract}

Index Terms-Geometrical calibration, optimization, septa-tocrystal position, slit-slat collimator, SPECT.

\section{INTRODUCTION}

$\mathbf{S}$ MALL animal SPECT is an essential tool for preclinical biomedical research [1]. To expand the availability of SPECT for biomedical research, we worked on developing

Manuscript received November 29, 2010; revised April 15, 2011 and July 05, 2011; accepted July 25, 2011. Date of publication September 15, 2011; date of current version October 12, 2011.This work was supported in part by the Roswell Park Alliance Foundation under Grant 62-2426-01, the Canadian Institutes of Health Research (CIHR) Grant MOP-86717, the Canada Foundation for Innovation (CFI), and the Specialized Research Fund for the Doctoral Program of Higher Education in China (SRFDP200800031071).

X. Deng and R. Yao are with the Department of Nuclear Medicine, State University of New York at Buffalo, Buffalo, NY 14217 USA (e-mail: rutaoyao@buffalo.edu).

T. Ma is with the Department of Engineering Physics, Tsinghua University, Beijing 100084, China.

$\mathrm{R}$. Lecomte is with the Sherbrooke Molecular Imaging Center, Centre Hospitalier Universitaire de Sherbrooke, Sherbrooke QC J1H 5NY, Canada and also with the Department of Nuclear Medicine and Radiobiology, Université de Sherbrooke, Sherbrooke, QC J1H 5N4, Canada.

Color versions of one or more of the figures in this paper are available online at http://ieeexplore.ieee.org.

Digital Object Identifier 10.1109/TNS.2011.2163418
SPECT imaging capability by adding a collimator to an existing animal PET scanner. The benefits of this endeavor include lower overall cost for a PET/SPECT dual function system and potential PET/SPECT dual tracer applications. To achieve optimal performance in resolution and sensitivity on a SPECT system, it is critical that the system's geometrical parameters [2]-[5] used for image generation are accurate. Erroneous values would introduce artifacts and resolution loss [6], [7].

Slit-slat collimator [8]-[10] has the useful features of both pinhole and parallel hole collimators. It offers magnification in transaxial planes, large field-of-view (FOV) in the axial direction, and simple rotation scan protocol for distortion-free volume imaging. The animal SPECT system in this study used a slit-slat collimator inside the gantry of an animal PET scanner [11].

In this study, the slit-slat collimator consisted of a tungsten cylinder grooved with knife-edged slit-openings and multiple annular shaped slats stacked over the cylinder's axial axis. The detectors of the PET scanner, like other PET systems, were composed of pixelated crystals. This particular collimator-detector setup is defined by a set of geometrical parameters, namely slit-aperture position (SAP), slat-to-crystal position (SCP), slat obliqueness and center-of-rotation (COR). COR is to account for the fact that a partial or complete rotation of the subject may be involved in tomographic imaging on the SPECT [12].

The transaxial and axial resolution are determined by the slit and slat components, respectively. Most of the geometry parameters can be optimized based on the well-established pinhole and parallel hole theory for conventional Anger logic detectors [13]. But due to the combination of slat and pixelated detector crystals, the SCP is a new factor on system response with respect to those for conventional continuous detectors. We first observed the impact of SCP in one of our previous studies [14]. With a fixed setup of the slit-slat collimator, the full width at half maximum (FWHM) of axial point spread function (PSF) showed a periodical variation pattern. This pattern does not exist on conventional continuous detectors. To accurately model the system response and therefore improve the reconstructed image quality in axial direction, we need to develop a method to optimize and determine the SCP for the SPECT system with slat insert. This has not been reported in the published literatures. As the SCP mainly changes the PSF in axial direction and has negligible effect on transaxial performance, only the dependency of axial resolution on SCP has been investigated in this study.

In this work, we first studied the impact of SCP on overall system axial resolution. This is most relevant for identifying 


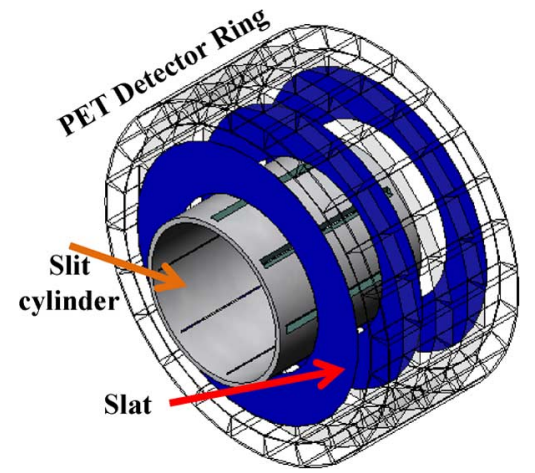

Fig. 1. The setup of having a slit-slat collimator insert inside an animal PET scanner for SPECT imaging is illustrated in the diagram. Only three slats are shown for clarity.

an optimized configuration at the system design stage. Then we present a calibration method to determine the accurate value of SCP after the slit-slat collimator was placed inside the detector gantry for SPECT imaging.

\section{MATERIALS AND METHODS}

\section{A. Animal PET Scanner and the Slit-Slat Collimator}

The animal PET scanner used in this study was a microPET Focus-120 system (Siemens Preclinical Solutions, Knoxville, TN). It consisted of 4 detector rings with a $14.7 \mathrm{~cm}$ diameter and $7.6 \mathrm{~cm}$ axial extent [15]. Each detector ring had 24 detector blocks. Each block consisted of a $12 \times 12$ array of Lutetium Oxyorthosilicate (LSO) crystals and each crystal had a size of $1.6 \times 1.6 \times 10 \mathrm{~mm}^{3}$.

Fig. 1 shows a PET detector ring with a slit-slat collimator placed inside. The slit-slat collimator consisted of a slit-cylinder (cylinder grooved with slits) and annular shaped plates (slats). Both the cylinder and the slats were made of tungsten. Eight slit-apertures were uniformly grooved on the cylinder along the cylinder's axial direction. There were a total of 97 slats covering an axial field-of-view of $76 \mathrm{~mm}$ (only 3 slats are shown in the figure), and a transaxial FOV of $50 \mathrm{~mm}$ diameter. The wall thickness of the slit cylinder was $2.5 \mathrm{~mm}$. The thickness of the slat was $0.2 \mathrm{~mm}$. Spacers with $0.6 \mathrm{~mm}$ thickness and same annular shape were placed between the neighboring slats. The spacer material is Rohacell (EVONIK Inc., USA), which has a density of $0.052 \mathrm{gram} / \mathrm{cm}^{3}$ and compressive strength of $0.9 \mathrm{MPa}$. The slit-apertures' open-angle was 85 degrees and the slit-opening width $w$ was $0.6 \mathrm{~mm}$. The inner and outer diameters of the slat were 84 and $132 \mathrm{~mm}$, respectively. The centers of the slat were aligned to the axial axis of the slit-cylinder and the animal PET detector ring.

In this study, imaging objects were rotated for tomographic imaging. We are aware that this is undesired for in vivo studies and have explored alternative approaches [12]. The validity of the optimization and calibration method investigated should not be affected by the tomographic scheme used.

\section{B. Axial PSF With Pixelated Detector Crystals}

The analytical PSF model for conventional SPECT system with continuous detectors, with the consideration of geometric response, photon penetration path in detector crystal, and scatter effect of collimator, has been studied by several groups [16]-[20]. However, it is not straightforward to extend the existing analytical model to a SPECT system with pixelated detectors. In this study, we calculated the axial PSF based on geometrical solid angle only. The other factors, such as the detector efficiency of the pixelated crystals were assumed to be secondary and neglectable.

Fig. 2(A) shows the photon flux emitted by a point source at position $P$ that reaches the detector through the opening formed by gap between the $k^{\text {th }}$ slat-gap and a slit-aperture. The $k^{\text {th }}$ slat-gap is between the $k^{\text {th }}$ and $(k+1)^{\text {th }}$ slat. The descriptions of all the variables illustrated in Fig. 2 and used in the following derivations are shown in Table I.

The photon exposure area $S$ on the $m^{\text {th }}$ crystal corresponds to the solid angle $\Omega_{m, k}$ subtended at $P$. The number of photons collected by the $m^{\text {th }}$ crystal $p_{m}$ is

$$
p_{m}=A_{0} \sum_{k} \frac{\Omega_{m, k}}{4 \pi},
$$

where, $p_{m}$ is the axial PSF model for the slit-slat SPECT with pixelated detector crystals. Assuming the projection area $S$ is a parallelogram, $\Omega_{m, k}$ is the surface integral over the area $S$, [See equation (2) at bottom of next page] where, $\alpha_{m, k, s t a r t}$ and $\alpha_{m, k, e n d}$ are the starting and ending out-of-plane angle of the exposure area $S$ to the point $P$. The corresponding exposure points of $\alpha_{m, k, s t a r t}$ and $\alpha_{m, k, e n d}$ on the crystals are $z_{m, k, s t a r t}$ and $\mathrm{z}_{m, k, e n d}$, as shown in Fig. 2(C). Equation (2) is exact if $P$ is located on the $Z$-axis. It is an approximation if $P$ is off the line passing SAP and $O$.

The in-plane angle $\gamma_{s}$ of $S$ is

$$
\gamma_{\mathrm{s}} \approx 2 \operatorname{atan}(w / 2 h)
$$

So

$\Omega_{m, k}=\left\{\begin{array}{cl}\gamma_{s}\left[\frac{z_{m, k, \text { start }}-z_{p}}{\sqrt{\left(z_{m, k, \text { star. }}-z_{p}\right)^{2}+H^{2}}}\right. \\ \left.-\frac{z_{m, k, \text { end }}-z_{p}}{\sqrt{\left(z_{m, k, \text { start }}-z_{p}\right)^{2}+H^{2}}}\right] & , z_{m, k, \text { start }}<z_{m, k, \text { end }} \\ 0, \quad & , z_{m, k, \text { start }} \geq z_{m, k, \text { end }}\end{array}\right.$

where

$$
\begin{aligned}
& z_{m, k, \text { start }}=\max \left[\frac{H}{h+g}\left(z_{k}+\frac{t}{2}\right),\right.\left.\frac{H}{h+g+a}\left(z_{k}+\frac{t}{2}\right), z_{m}-\frac{w_{\mathrm{c}}}{2}\right], \\
& z_{m, k, \text { end }}=\min \left[\frac{H}{h+g}\left(z_{k}+\frac{t}{2}+d\right),\right. \\
&\left.\frac{H}{h+g+a}\left(z_{k}+\frac{t}{2}+d\right), z_{m}+\frac{w_{\mathrm{c}}}{2}\right],
\end{aligned}
$$

and $z_{k}=\Delta z+k *(d+t)$.

\section{SCP Impact on PSF Width $(\overline{R M S})$}

Usually when there are three or more sampling points above the PSF's half-maximum level, FWHM is used as the width 


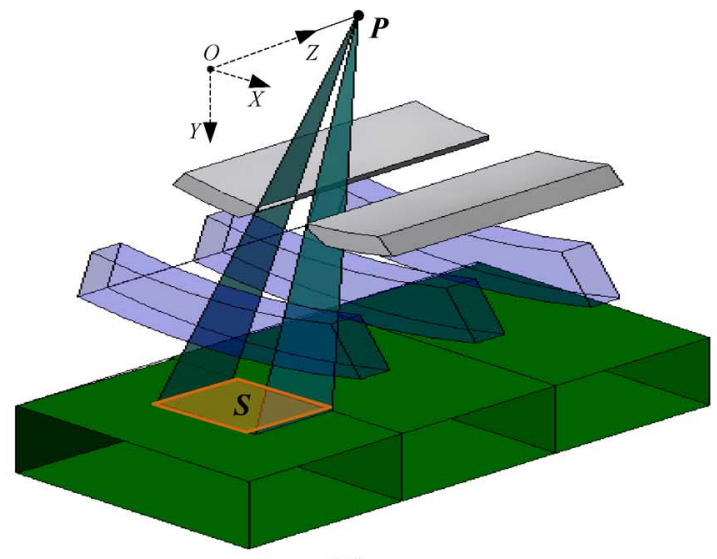

(A)

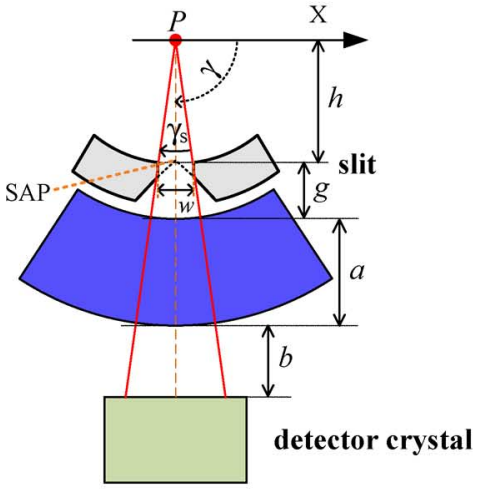

(B)

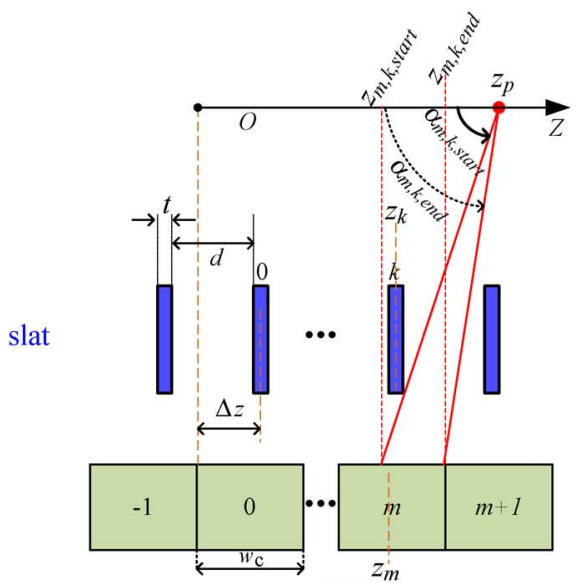

(C)

Fig. 2. Illustration of the slit-slat collimator and the variables used for derivation of the axial PSF model. (A) Overall view of a point source at $P$ projects to an area $S$ of crystals on the detector ring. (B) The transaxial section view passing point $P$. (C) The axial sectional view passing $P . O-X Y Z$ is the reference frame (Cartesian coordinate) of the imaging system, and the origin $O$ is the center of field of view (CFOV).

measure of the PSF and can be obtained by a Gaussian fitting with acceptable accuracy. Due to the system's high resolution design of $1.5 \mathrm{~mm}$ FWHM and relatively large size $(1.6 \mathrm{~mm})$ of the pixilated detector crystals, $95 \%$ of the PSFs had less than two sampling points (i.e. two crystals) above the PSF's half-maximum level. So instead of using FWHM, the root mean square (RMS) width was chosen for measuring the axial PSF spread width [21]. For a point source at $z_{p}$,

$\operatorname{RMS}_{\mathrm{AX}}\left(\Delta z, z_{p}\right)=\sqrt{\sum_{m=m_{0}}^{m_{0}+M-1} p_{m}\left(z_{m}-\bar{z}\right)^{2} / \sum_{m=m_{0}}^{m_{0}+M-1} p_{m}}$

where $\bar{z}$ is the centroid of the PSF, $m_{0}$ is the start crystal index of the PSF, $m$ is crystal index, and $M$ is the total number of crystals that the PSF spreads over in the axial direction. For the current design, $M=9$ was chosen, i.e. all the PSFs spread over less than 9 crystals. $\bar{z}$ is calculated by

$$
\bar{z}=\sum_{m=m_{0}}^{m_{0}+M-1} p_{m} z_{m} / \sum_{m=m_{0}}^{m_{0}+M-1} p_{m}
$$

To assess the impact of SCP, the average RMS width $(\overline{R M S})$ was calculated as the overall measure of spatial resolution,

$$
\overline{\operatorname{RMS}}_{\mathrm{AX}}(\Delta z)=(1 / Q) \sum_{q=0}^{Q-1} \operatorname{RMS}_{\mathrm{AX}}\left(\Delta z, z_{q}\right)
$$

where $z_{q}$ denotes the uniformly distributed point source positions over an axial symmetric cycle $z_{\text {cyc }}$ and $Q$ is the total number of sample positions evaluated. In the SPECT system

$$
\Omega_{m, k}= \begin{cases}\iint_{s} \sin \alpha d \gamma d \alpha & \\ \approx \gamma_{s}\left[\cos \left(\alpha_{m, k, s t a r t}\right)-\cos \left(\alpha_{m, k, \text { end }}\right)\right], & \alpha_{m, k, \text { start }}<\alpha_{m, k, \text { end }} \\ 0, & \alpha_{m, k, \text { start }} \geq \alpha_{m, k, \text { end }}\end{cases}
$$


TABLE I

The Description of the VARIABLES ILlustrated IN Fig. 2

\begin{tabular}{|c|c|}
\hline Symbol & Name (value used in the current study) \\
\hline$a$ & radial height of slat $(24.0 \mathrm{~mm})$ \\
\hline$A_{0}$ & $\begin{array}{l}\text { total number of emitted photons from point source } \\
\text { (product of source activity and acquisition time) }\end{array}$ \\
\hline$b$ & radial gap between slat outer ring and detector ring $(7.6 \mathrm{~mm})$ \\
\hline$d$ & slat gap or the spacer thickness $(0.6 \mathrm{~mm})$ \\
\hline$f$ & the focal length of the slit $(f=g+a+b=36.6 \mathrm{~mm})$ \\
\hline$g$ & radial gap between slit and inner ring of the slat $(5.0 \mathrm{~mm})$ \\
\hline$h$ & slit radius $(37.0 \mathrm{~mm})$ \\
\hline$H$ & the distance between point source and detector $(H=h+f=73.6)$ \\
\hline$P$ & Point source position (referring to the imaging system) \\
\hline$S$ & The area that $\mathrm{P}$ shines through a slit-slat opening on detector \\
\hline$t$ & slat thickness $(0.2 \mathrm{~mm})$ \\
\hline$w$ & slit opening width $(0.6 \mathrm{~mm})$ \\
\hline$w_{\mathrm{c}}$ & crystal width $(1.6 \mathrm{~mm})$ \\
\hline$z_{k}$ & $\begin{array}{l}\text { the axial center position of the } k^{\text {th }} \text { slat } \\
\left(z_{k}=\Delta z+k^{*}(d+t)=\Delta z+k^{*} 0.8 \mathrm{~mm}\right)\end{array}$ \\
\hline$z_{m}$ & $\begin{array}{l}\text { the axial center position of the } m^{\text {th }} \text { crystal } \\
\left(z_{m}=(m+0.5) * 1.6 \mathrm{~mm}\right)\end{array}$ \\
\hline$z_{\mathrm{p}}$ & axial position of point source $(-37.0 \mathrm{~mm}$ to $37.0 \mathrm{~mm})$ \\
\hline$\beta$ & slit open angle ( 85 degree) \\
\hline$\gamma$ & $\begin{array}{l}\text { Oblique projection angle in the transverse view } \\
\text { (due to the relative position of } P \text { and } S \text { ) }\end{array}$ \\
\hline$\gamma_{s}$ & $\begin{array}{l}\text { slit opening angle to the point source } \\
\text { (due to the relative position of } P \text { and slit opening) }\end{array}$ \\
\hline$\Delta z$ & $\begin{array}{l}\text { SCP, which is defined as the distance between the line passing } \\
\text { through the middle of the } 0^{\text {th }} \text { slat and the starting edge of the } 0^{\text {th }} \\
\text { crystal }(0 \text { to }(d+t) \text {, i.e., } 0 \text { to } 0.8 \mathrm{~mm})\end{array}$ \\
\hline
\end{tabular}

studied, the slat and pixelated crystals were uniformly distributed over the axial direction with a cycle of $0.8 \mathrm{~mm}$ and 1.6 $\mathrm{mm}$, respectively. Therefore, the relative position of the slat to the most adjacent crystal would repeat in a cycle of $1.6 \mathrm{~mm}$.

\section{Optimization of $S C P$}

To search for the SCP that optimizes axial resolution and sensitivity, the collimator-insert was shifted over a series of SCP positions in the axial direction. The range of SCP was from $0.00 \mathrm{~mm}$ to $0.80 \mathrm{~mm}$ and the step size was $0.04 \mathrm{~mm}$. At each $\mathrm{SCP}, \overline{R M S}$ and average sensitivity were calculated from 32 point source positions uniformly sampled over a $3.2 \mathrm{~mm}$ range (two cycles) along the central axis of the detector ring. This evaluation was performed with both analytical calculation (Section II-B) and Monte Carlo simulated data.

\section{E. PSF-Based SCP Calibration}

We found that the centroid of PSF was insensitive to SCP variations and not suitable for SCP calibration. So we developed a SCP calibration technique that uses the full shape of PSF. The implementation of this technique is as follows.

A series of projections are obtained by scanning a point source in axial direction starting from the position $z_{p 0}$. SCP, i.e. $\Delta z$, is estimated by minimizing the least-square cost function

$$
F(\Gamma)=\frac{1}{2} \sum_{i=1}^{N} \sum_{m=m_{0, i}}^{m_{0, i}+M-1}\left(p_{i, m}(\Gamma)-c_{i, m}\right)^{2} .
$$

TABLE II

The KNOWn PARAmeters' VAlue and ERror Range

\begin{tabular}{|c|c|c|c|}
\hline Symbol & Name & Ideal Value & Error range \\
\hline$a$ & radial height of slat & $24.0 \mathrm{~mm}$ & $\pm 0.1 \mathrm{~mm}$ \\
\hline$b$ & gap of slat and detector & $7.80 \mathrm{~mm}$ & $\pm 0.10 \mathrm{~mm}$ \\
\hline$d$ & slat gap & $0.60 \mathrm{~mm}$ & $\pm 0.02 \mathrm{~mm}$ \\
\hline$g$ & gap of slit and slat & $5.0 \mathrm{~mm}$ & $\pm 0.1 \mathrm{~mm}$ \\
\hline$h$ & CFOV to slit height & $37.0 \mathrm{~mm}$ & $\pm 0.05 \mathrm{~mm}$ \\
\hline$t$ & slat thickness & $0.20 \mathrm{~mm}$ & $\pm 0.02 \mathrm{~mm}$ \\
\hline$w$ & slit width & $0.60 \mathrm{~mm}$ & $\pm 0.05 \mathrm{~mm}$ \\
\hline$w_{\mathrm{c}}$ & crystal width & $1.60 \mathrm{~mm}$ & $\pm 0.01 \mathrm{~mm}$ \\
\hline$z_{\mathfrak{p}}$ & point source axial position & $0.00 \mathrm{~mm}$ & $\pm 0.04 \mathrm{~mm}$ \\
\hline$\beta$ & slit open angle & $85.0 \mathrm{deg}$ & $\pm 0.1 \mathrm{deg}$ \\
\hline
\end{tabular}

where $\Gamma=\left\{\Delta z, A_{0}, z_{p 0}\right\}$ is the parameter set to be estimated, $N$ is the total number of projections, $i=0,1, \ldots, N-1$ is the projection index, $m_{0, i}$ is the start crystal index of the $i^{\text {th }}$ axial PSF (axial PSF in the $i^{\text {th }}$ projection), and $p_{i, m}$ and $c_{i, m}$ are the predicted and measured counts of the $m^{\text {th }}$ crystal in the $i^{\text {th }}$ axial PSF. The point source activity $A_{0}$ and the initial position $z_{p 0}$ are included in $\Gamma$ as they vary for every measurement. Other parameters that have known values from manufacture or measurements are not included in $\Gamma$. Table II show these known parameters and their error ranges. Here the inaccuracies are from the fabricating process and relatively small to be neglected for the axial PSF shape. The Levenberg-Marquardt algorithm is used to solve for $\Gamma$.

\section{F. SVD Analysis: The Uniqueness and Accuracy of the Calibration Method}

To validate the uniqueness and accuracy of the SCP calibration method, a SVD-based method [4] was used. The method applies SVD to the Jacobian matrix $\mathbf{J}$ of the cost function in (9),

$$
\mathbf{J}=\mathbf{U S} \mathbf{V}^{T}=\sum_{j=1}^{r} s_{j} \mathbf{u}_{j} \mathbf{v}_{j}^{T},
$$

where, $r$ is the number of parameters to be estimated, $\left\{\mathrm{u}_{i}, i=\right.$ $1, \ldots, N\}$ and $\left\{\mathrm{v}_{j}, j=1, \ldots, r\right\}$ are the left-singular and rightsingular vectors respectively, and $s_{1} \geq s_{2} \geq \ldots \geq s_{r}$ are the singular values. The uniqueness of the calibration was identified by assessing the non-singularity of the Jacobian matrix, i.e., the calibration is unique if all the singular values are not zero. The calibration accuracy, $\sigma_{\Gamma}$, was quantified by analyzing the SVD components,

$$
\sigma_{\Gamma_{j}}^{2}=\left[\left(\mathbf{J}^{\mathrm{T}} \mathbf{J}\right)^{-1} \mathbf{J}^{\mathrm{T}} \operatorname{diag}\left\{\sigma_{1}^{2}, \sigma_{2}^{2}, \cdots, \sigma_{n}^{2}\right\} \mathbf{J}\left(\mathbf{J}^{\mathrm{T}} \mathbf{J}\right)^{-1}\right]_{j, j},
$$

where $\sigma_{1}^{2}, \sigma_{2}^{2}, \ldots \sigma_{n}^{2}$, are the measurement variance of counts in crystals. Here, the $\sigma_{\mathrm{j}}$ is considered as the square root of the measured counts, i.e., $\sigma_{j}=\sqrt{c_{j}}$.

For the SVD analysis of the uniqueness and accuracy, 120 parameter sets of $\Gamma$ were obtained by permutations of $A_{0}=$ $10^{8}, 10^{9}$ and $10^{10}, z_{p 0}=-1.8$ to $-1.4 \mathrm{~mm}$ with a step size of $0.1 \mathrm{~mm}$, and SCP $=0.0 \mathrm{~mm}$ to $0.7 \mathrm{~mm}$ with a step size of 0.1 $\mathrm{mm}$. The other parameters used are shown in Table II. For each 


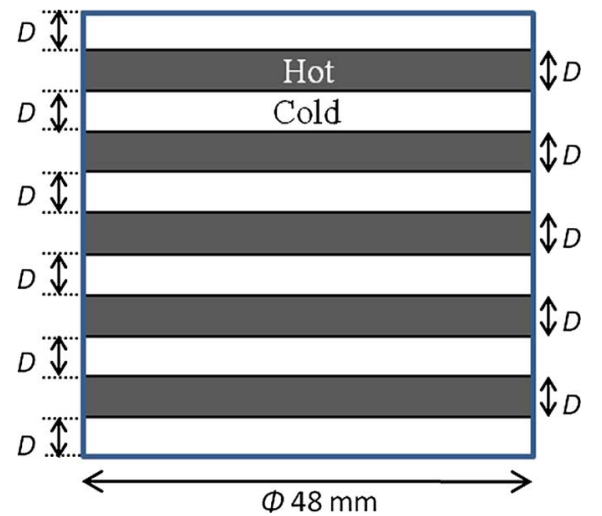

Fig. 3. The cross sectional view of an axial resolution phantom. The hot discs (in gray) were filled with ${ }^{99 \mathrm{~m}} \mathrm{Tc}$ solution. The gaps between hot discs were left empty. The thicknesses of both the discs and the gaps are $D$ for each phantom. Four phantoms, with $D=1.0,1.2,1.4$ and $1.6 \mathrm{~mm}$, were studied.

$\Gamma$, the SVD components $U, S$ and $V$ of $J$ were calculated by using (10), (11).

\section{G. SCP Calibration With MC Simulated Data}

A total of $162 \mathrm{MC}$ simulation studies, each corresponds to a geometrical setting, were performed to study the proposed SCP calibration method. The parameter settings were obtained from permutations of $A_{0}=0.95 \times 10^{9}, 1.0 \times 10^{9}, 1.05 \times 10^{9}$; $z_{p 0}=-1.64,-1.6,-1.56 \mathrm{~mm}, d=0.58,0.60,0.62 \mathrm{~mm}$, $t=0.18,0.20,0.22 \mathrm{~mm}$, and $\Delta z=0.0,0.4 \mathrm{~mm}$. The $3 \mathrm{op}-$ tions of each parameter were derived from Table II: the lower end of error range, the ideal value, and the higher end of error range. The point source activity $A_{0}$ was assumed to be known from dose calibrator measurement with an error range of $\pm 5 \%$. Other parameters in the parameter sets used in the simulation are the ideal values shown in Table II. For each parameter set, 32 projections of a ${ }^{57} \mathrm{Co}$ point source were generated with the GATE MC simulation package [22]. Here the choice of 32 projections was based on our previous study on calibration accuracy and projection numbers [4]. Other parameters used were $A_{0}=1.0 \times 10^{9}, z_{p 0}=-1.6 \mathrm{~mm}$.

For each simulated study, SCP was determined by solving the (9) with the 32 simulated projections.

\section{H. SCP Impacts on Phantom Images}

To demonstrate the effect of SCP position and the calibration accuracy of SCP on the axial imaging resolution, a series of phantoms with axial structural patterns were simulated. Each phantom consisted of interleaved hot and cold discs with the same thicknesses and separation $D$, as shown in Fig. 3. A total of 4 phantoms were simulated with disc thickness $D$ incrementing from 1.0 to $1.6 \mathrm{~mm}$ with a step size of $0.2 \mathrm{~mm}$. The hot discs of the phantoms were ${ }^{99 \mathrm{~m}} \mathrm{Tc}$ source with activity concentration of $5.43 \mathrm{MBq} / \mathrm{cm}^{3}$. Each phantom was scanned at 120 rotational steps and 3 degree per step. To average out the axial resolution non-uniformity, the phantom was also moved $0.04 \mathrm{~mm}$ in axial direction after every 3 steps. An OSEM iterative reconstruction program with 8 subsets and 10 iterations was used for the image reconstruction. The image voxel size was $0.5 \mathrm{~mm} \times 0.5 \mathrm{~mm} \times$ $0.2 \mathrm{~mm}$.
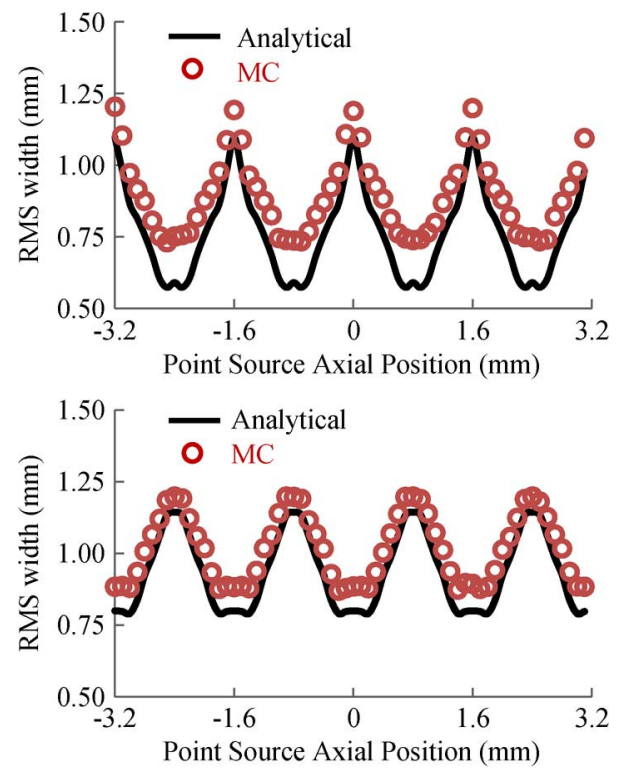

Fig. 4. The RMS width of an axial PSF varies as the point's axial location changes. The patterns of variation and discrepancy between analytical and MC results are different for $\mathrm{SCP}=0.0 \mathrm{~mm}$ (top) and for $\mathrm{SCP}=0.4 \mathrm{~mm}$ (bottom).

To assess the impact of SCP optimization, studies of the phantoms described above were simulated with five SCP positions, $\mathrm{SCP}=0.0 \mathrm{~mm}$ to $0.4 \mathrm{~mm}$ with a step size of $0.1 \mathrm{~mm}$. The reconstructed images, with perfect knowledge of SCP, were compared. The peak-to-valley ratio of the disc was used to evaluate the SCP optimization.

To assess the impact of SCP calibration, studies of the phantoms simulated with $\mathrm{SCP}=0.0 \mathrm{~mm}$ were reconstructed with 3 system matrices. The 3 system matrices were derived with SCP $=0.0 \mathrm{~mm}, 0.04 \mathrm{~mm}$ and $0.4 \mathrm{~mm}$, which correspond to perfect calibration, currently achieved SCP accuracy, and maximum SCP discrepancy, respectively.

\section{RESULTS}

\section{A. Optimization of SCP}

Fig. 4 shows the variation of axial PSF in terms of RMS width along the central axial axis. The RMSs calculated from the analytically modeled and the MC simulated PSFs match in cyclic period and phase for both SCP $=0.0 \mathrm{~mm}$ (top) and $0.4 \mathrm{~mm}$ (bottom). The matchings serve as a validation of the analytical model. The discrepancy of results between the analytical modeled and the MC simulation data is likely due to simplification of the analytical model - photon penetration effect is not taken into account. Similar discrepancy is also observed in Fig. 5.

Fig. 5 shows the dependency of average sensitivity and axial PSF's $\overline{R M S}$ on SCP. This is because the gaps between pixelated crystals on the system studied was negligibly small $(<5 \%$ of the crystal pitch), so SCP had little effect on the total photon flux received by the crystals. So the average sensitivity remains almost constant as SCP varies.

Since photon penetrations of collimator were not considered in the analytical calculation, the integration of the corresponding PSFs and therefore the average sensitivity calculated is smaller 


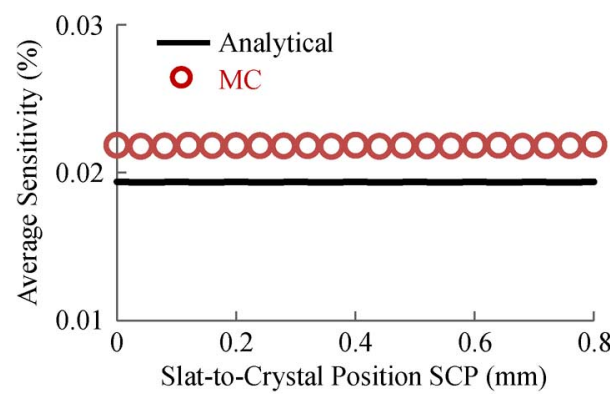

TABLE III

The Singular Vectors (v1, ., v3) (Row 2 to 4) and Singular Values $\mathrm{s}_{\mathrm{j}}$ (LAst Row) For the Calibration With 1 Point Source, 1 Slit and 32 Projections. Column 1 Shows the NAMe of EAch Parameter, AND THE Last Column is the CorResponding STD. The KnOwn Parameter Set $\Gamma$ WAS $\left\{A_{0}, \mathrm{z}_{\mathrm{p} 0}, \Delta z\right\}=\left\{10^{9},-1.6 \mathrm{~mm}, 0.0 \mathrm{~mm}\right\}$

\begin{tabular}{ccccc}
\hline \hline Parameter & $\mathrm{v}_{1}$ & $\mathrm{v}_{2}$ & $\mathrm{v}_{3}$ & $\mathrm{STD}$ \\
\hline$A_{0}$ & 0.0000 & 0.0000 & -1.0000 & $1.1977 \mathrm{E}-13$ \\
$z_{\mathrm{p} 0}$ & -0.4312 & -0.9022 & 0.0000 & $0.0011 \mathrm{~mm}$ \\
$\Delta z$ & 0.9022 & 0.4312 & 0.0000 & $0.0006 \mathrm{~mm}$ \\
$s_{\mathrm{j}}$ & $2.3934 \mathrm{E} 5$ & $4.9728 \mathrm{E} 4$ & $1.0575 \mathrm{E}-4$ & \\
\hline \hline
\end{tabular}

variance of other parameters in Table II was not considered in the SVD analysis results (Table III), and this may have caused the standard deviation difference of SCP between the SVD analysis and Monte Carlo simulation.

\section{SCP Impacts on Phantom Images}

Fig. 5. Average sensitivity (top) and $\overline{R M S}$ (bottom) as a function of SCP from analytically calculated and Monte Carlo simulated axial PSFs.

than its counterpart from MC simulation, as shown in Fig. 5 (top). Also, the slat-edge penetration spreads PSFs wider as shown in Fig. 5 (bottom).

The analytically calculated resolution in terms of RMS width and average RMS width shows varied degrees of agreement with the corresponding MC simulation results, as shown in Fig. 4 and Fig. 5. The $\overline{R M S}$ changes in the range of $0.90 \mathrm{~mm}$ to $1.05 \mathrm{~mm}$ for analytically modeled PSFs and $0.75 \mathrm{~mm}$ to $0.95 \mathrm{~mm}$ for $\mathrm{MC}$ simulation, i.e., about $17 \%$ variation as SCP changes. $\overline{R M S}$ is at its minimum value when $\mathrm{SCP}=0.0 \mathrm{~mm}$ and at its maximum when $\mathrm{SCP}=0.4 \mathrm{~mm}$. That is, if the slat is aligned to the crystal edge in axial direction $(\mathrm{SCP}=0.0 \mathrm{~mm})$, optimal axial resolution is obtained.

\section{B. SVD Analysis of the Calibration Method}

The minimal singular value for the numerical simulations of the 120 parameter sets was $9.0636 \times 10^{-5}$. All the singular values were always non-zero. This means that the proposed calibration method has a unique solution. Table III shows the singular values and right singular vectors calculated from the numerically simulated projections of one parameter set. The standard deviation of SCP is $0.0006 \mathrm{~mm}$.

\section{SCP Calibration With MC Simulated Data}

The 162 parameter sets used in Monte Carlo simulation for calibration test consisted of 81 sets with SCP $=0.0 \mathrm{~mm}$ and 81 sets with $\mathrm{SCP}=0.4 \mathrm{~mm}$. For the tests with $\mathrm{SCP}=0.0 \mathrm{~mm}$, the estimated SCP varied from -0.03 to $0.03 \mathrm{~mm}$ with mean and standard deviation values of $0.00 \pm 0.01 \mathrm{~mm}$. For the tests with SCP $=0.4 \mathrm{~mm}$, the estimated SCP varied from 0.37 to $0.43 \mathrm{~mm}$ with mean and standard deviation values of $0.40 \pm$ $0.01 \mathrm{~mm}$. The error of the SCP calibration was less than 0.04 $\mathrm{mm}$, which was expected to be adequate for a SPECT system with $\sim 1 \mathrm{~mm}$ axial image resolution. It must be noted that the

Fig. 6 shows the coronal sectional view of a series of digital phantoms (left column), and the corresponding reconstructed images with optimal SCP $(0.0 \mathrm{~mm}$, middle column $)$ and least optimal SCP $(0.4 \mathrm{~mm}$, right column)setup. For each system setup with a given $\mathrm{SCP}$, the corresponding system matrix was derived with the same SCP value. From top row to bottom row, the disk thickness $D$ of the corresponding phantom (see also the illustration in Fig. 3) is $1.6 \mathrm{~mm}, 1.4 \mathrm{~mm}, 1.2 \mathrm{~mm}$ and $1.0 \mathrm{~mm}$, respectively. Fig. 7 shows the phantom images' section profiles from the location indicated by the lines overlaid on the images in Fig. 6. Fig. 8 shows the peak-to-valley ratio of the center disk at $z=0 \mathrm{~mm}$ from phantom images with $D=1.6 \mathrm{~mm}$ and different SCP values. Both the images and profiles show that a visible separation of the hot disc with $1.0 \mathrm{~mm}$ thickness can be achieved with either the optimized or the non-optimized SCP setup. But it can be seen in Figs. 7 and 8 that the optimal SCP achieves better image quality in the axial direction, as indicated by the higher peak-to-valley ratio. This is especially clear for the phantom images with larger disk size, e.g., for the phantom with $D=1.6 \mathrm{~mm}$ (1st row), the peak-to-valley ratio degrades from 25 to 2 for the optimal SCP $(0.0 \mathrm{~mm})$ and least optimal SCP $(0.4 \mathrm{~mm})$, respectively.

The SCP optimization, however, shows less improvement on image quality as the disk size decreases. For disk $D=1.0 \mathrm{~mm}$, the optimal SCP $(0.0 \mathrm{~mm})$ doesn't have significant effect on image quality. The smaller disk size is close to the system resolution limit, and the SCP impact on image quality was concealed by the effect of system's finite resolution.

Fig. 9 shows the effect of SCP calibration accuracy. The coronal views of a series of the simulated axial resolution phantoms reconstructed with system matrices derived from matching and mismatching SCP values are compared. The system setup used the optimal SCP $(0.0 \mathrm{~mm})$. From left to right, the 1st column presents the original digital phantoms. The 2nd column shows the reconstructed images using the system matrix derived with the perfectly calibrated SCP $(0.0$ $\mathrm{mm}$ ). Respectively, the system matrices used for reconstructing 


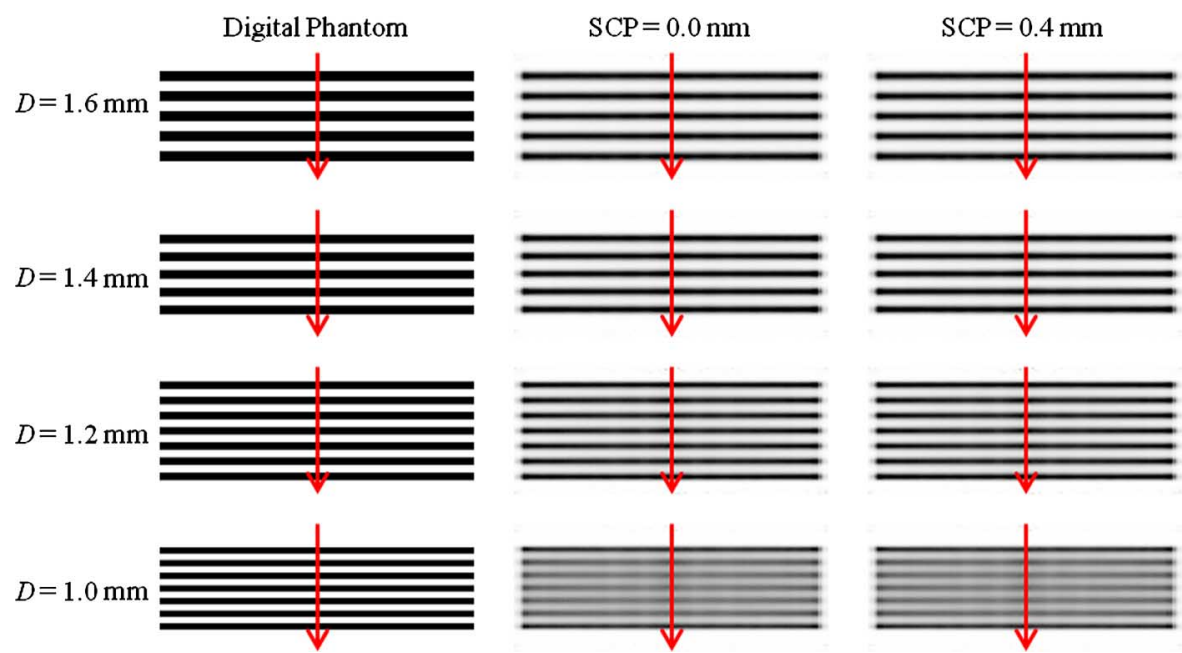

Fig. 6. Effect of SCP optimization: From top to bottom, the rows correspond to the coronal sectional views of axial phantoms with $D=1.6 \mathrm{~mm}, 1.4 \mathrm{~mm}$, $1.2 \mathrm{~mm}$ and $1.0 \mathrm{~mm}$, respectively. From left to right, the columns corresponds to the original digital phantom (left), the images with optimal SCP $=0.0 \mathrm{~mm}$ (middle), and the images with least optimal $\mathrm{SCP}=0.4 \mathrm{~mm}$ (right).
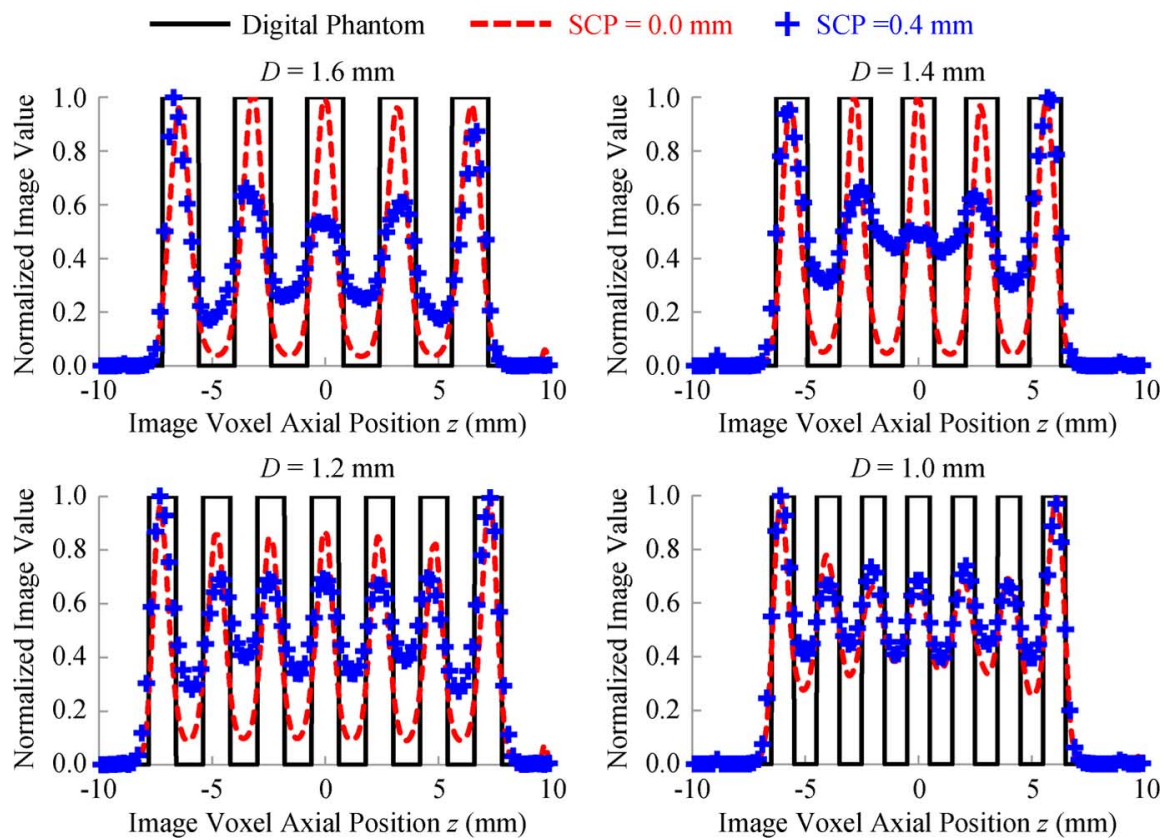

Fig. 7. The sectional profiles obtained from the coronal sectional views of the phantoms $D=1.6 \mathrm{~mm}, 1.4 \mathrm{~mm}, 1.2 \mathrm{~mm}$, and $1.0 \mathrm{~mm}$ in Fig. 6 . The profile locations are indicated by the lines overlaid on the images in Fig. 6.

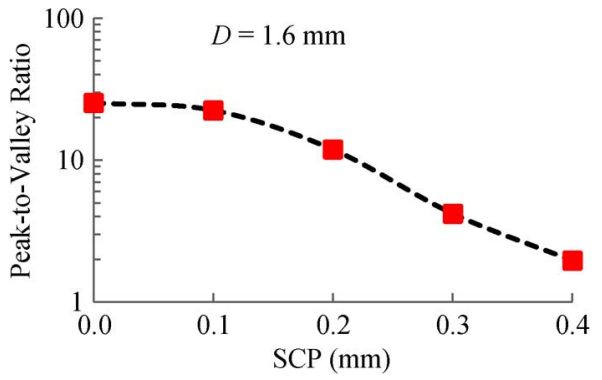

Fig. 8. Peak-to-valley ratio of the center disk at $z=0$ from the phantom with $D=1.6 \mathrm{~mm}$ and different SCP values.

the images in the 3rd and 4th columns were derived with SCP $=$ $0.04 \mathrm{~mm}$, which was the maximum error of SCP calibration, and SCP $=0.4 \mathrm{~mm}$, which was the maximum error without SCP calibration. The images show that a clear separation of the hot disc with $1.0 \mathrm{~mm}$ thickness could be achieved by using the perfect calibration (bottom row, 2nd column) and the currently achieved SCP calibration error $(0.04 \mathrm{~mm}$, bottom row, 3 rd column). When the SCP with the maximum error $(0.4 \mathrm{~mm})$ was used, only the phantom with hot disc thickness of $1.6 \mathrm{~mm}$ was reconstructed properly, i.e. with the correct number of discs and the disc pattern was distinguishable. For all the 4 phantoms with different disk thickness $D$, the reconstruction images with the $0.04 \mathrm{~mm} \mathrm{SCP}$ calibration error are visually identical to that obtained with the perfect calibration. So the calibration accuracy is enough for the current slit-slat SPECT system to achieve a $1 \mathrm{~mm}$ image resolution in axial direction. 


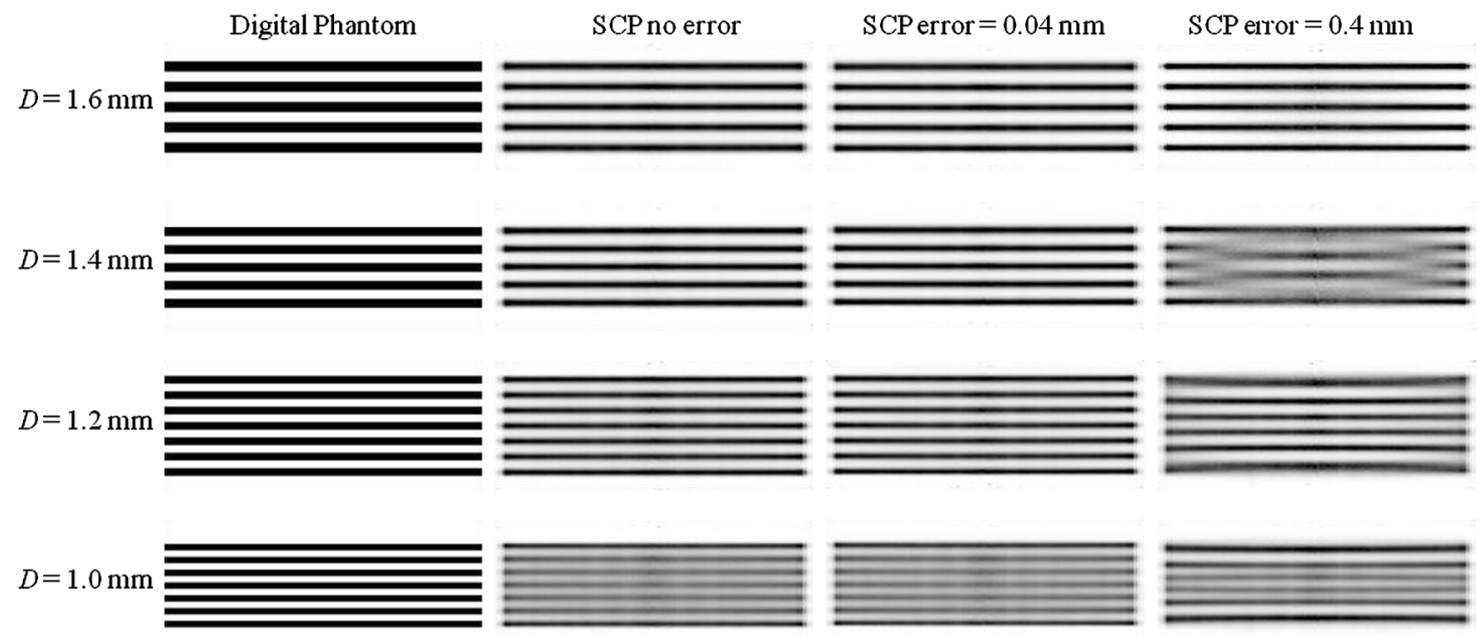

Fig. 9. Effect of SCP calibration error: From left to right, the columns correspond to the original digital phantom image (left) and the coronal sectional view of phantom images reconstructed with system matrices with perfect calibration, i.e. SCP $=0.0 \mathrm{~mm}$ (2nd column), with currently achieved SCP accuracy, i.e. $\mathrm{SCP}=0.04 \mathrm{~mm}$ (3rd column), and with maximal SCP discrepancy, i.e. $\mathrm{SCP}=0.4 \mathrm{~mm}$ (last column), respectively.

\section{DISCUSSION}

\section{A. The Dependency of SCP Optimization on Other Geometrical Parameters}

For the specific configuration of crystal size, slat thickness, gap between slats, and uniformly distributed crystals and slats, the SCP optimization study concluded that the central line of slat should be aligned to the crystal edge in axial direction to obtain the highest average axial resolution. However, any change to any of these factors will modify the pathways for the emitted photons to reach the crystals, and therefore change the magnitude and the distribution of the axial PSFs. As a result, the optimal SCP value will be different for achieving the highest axial resolution. Further, if the detector crystals are arranged non-uniformly in axial direction or the gaps between crystals are not neglectable, e.g., the LabPET-8 [23] consist of 32 crystal rings with alternating gaps 0.0 and $0.8 \mathrm{~mm}$ in axial direction, the choice of SCP will also impact sensitivity. So the optimization of SCP needs to take both resolution and sensitivity into account. The proposed approach can be easily extended to these different scenarios by adjusting the slat and scanner parameters in (1)-(5). It is especially useful for designing a system to achieve optimal system performance.

\section{B. What if the Crystal Pitch or Slat Pitch Changes?}

If crystal pitch or slat pitch or both are considered to be a variable, the proposed PSF model should be used to analyze the combined effect of SCP and crystal pitch. From what we have learned in this work, the classic resolution model proposed by Anger [17] for parallel hole collimator and Anger camera may not apply, due to the effect introduced by SCP. It is unclear, though, how different the result will be as compared to that of the classic model. This could be a direction for future studies.

\section{The Effect of Background Activity on Reconstructed Images}

In Figs. 6 and 7, the discs at the center of phantom images are significantly different from the discs at the ends. This can be explained by the dependency of convergence speed on background activity in OSEM reconstruction [24]. For any target area, its surrounding activity distribution is considered as background activity. So the activity in the adjacent hot discs of a disc is background which affects the reconstruction convergence speed of the disc. For the phantoms $D=1.2 \mathrm{~mm}$ and $D=1.0 \mathrm{~mm}$ with optimal SCP in Figs. 6 and 7, the discs in the center region were with background activity from two sides, and the discs at the ends were with background activity only from one side. That is, the background of the discs at the ends was lower than the discs at the center. Therefore, the discs at the ends were recovered faster than the center discs with the same iterations of OSEM reconstruction. And the discs at the ends were with higher recovery in terms of higher peak-to-valley ratios. This explains the difference in behavior of the discs at the center compared to the discs at the ends. For the phantoms $D=1.6 \mathrm{~mm}$ and $D=1.4 \mathrm{~mm}$ with optimal SCP, the gaps (equal to hot disc thickness $D$ ) between the hot discs were big enough to form an adequate "clearance", as compared to the PSF spread, around the discs at both center and ends of the phantom. That means the background activity was so low that the corresponding effect on the convergence of OSEM reconstruction could be neglected. Therefore all the hot discs at both center and ends were with the similar peak-to-valley ratio for phantom images $D=1.6 \mathrm{~mm}$ and $D=1.4 \mathrm{~mm}$ with optimal SCP.

\section{Usefulness of SCP Calibration}

For a system that is already built, SCP calibration may or may not be as useful. It depends on the approach selected to generate the system matrix. If the MC simulation or analytical calculation path is used for system matrix generation, then SCP calibration will be essential. If the system matrix is to be measured by scanning the FOV [25], SCP calibration may not be necessary. Ultimately, measuring the point spread functions by scanning a point source on the actual system can be the most accurate way to obtain the system matrix. However, this approach is constrained by: 1) the availability of an actual system; 2)the availability of a precise point source placement mechanism; and 3) the long scan time to acquire high-statistical data at all the grid 
points of the FOV. These constraints are prohibitive for a system in the development stage, in which case the actual scanner is either not existing physically or is frequently changed for performance optimization.

\section{E. Notable Techniques}

There are three technical details used in this work that we note here. First, we found that projection centroid is not sensitive to SCP changes. As the PSFs contain more complete information of projections, this work used a full-PSF based model to calibrate SCP. This is different from most of the publications on geometrical calibration [2], [4], [26]-[30], in which the centroid of measured and modeled projections was used in the calibration process. Second, RMS value was used to evaluate the axial resolution for optimization. Given the very limited sample points for each PSF, it is a useful evaluation index to estimate the collimator resolution and investigate the dependence of the PSFs on the system parameters. Compared to the commonly used index FWHM for PSF evaluation, RMS value can also contain some information outside the range of FWHM of PSFs, which may be more useful in the design stage. Third, the geometrical PSF derived for the slit-slat SPECT system was with pixelated detectors, which is different from what was published on geometrical sensitivity with continuous detectors [16], [19]. However, the PSF model can also be extended to be used for the cases of slit-slat or pinhole SPECT with continuous detectors. This can be done by changing either the scanner configuration parameters such as the crystal size, or the integration range of polar and azimuthal angles.

\section{CONCLUSION}

We developed a method to optimize and calibrate the slat-tocrystal position (SCP) in axial direction for a SPECT system with slit-slat collimator and pixelated crystals. As FWHM was not suitable for describing the spread of the PSFs with very limited sampling points, $\overline{R M S}$ was used as the evaluation index for the width of axial PSFs. SCP optimization was achieved by finding the SCP that provides the minimal $\overline{R M S}$ without loss of sensitivity. SCP calibration was based on the least-square-error method that minimizes the difference between the MC simulated and analytically modeled projections. Both the optimization and calibration techniques were evaluated with MC simulated data.

We showed that the $\overline{R M S}$ was $\sim 15 \%$ better for the optimal SCP as compared to that with the least-optimal SCP, and system sensitivity was not affected by SCP. The SCP error achieved by the proposed calibration method was less than $0.04 \mathrm{~mm}$. The reconstructed images of MC simulated phantoms showed expected resolution and were artifact free with the calibrated value. We conclude that the proposed optimization and calibration method is effective for the slit-slat collimator based SPECT systems.

\section{ACKNOWLEDGMENT}

This work utilized the high-performance computational capabilities of the Linux cluster at the Center for Computational Research, State University of New York at Buffalo.

\section{REFERENCES}

[1] S. R. Meikle, P. Kench, M. Kassiou, and R. B. Banati, "Small animal SPECT and its place in the matrix of molecular imaging technologies," Phys. Med. Biol., vol. 50, pp. 45-61, 2005.

[2] G. T. Gullberg, B. M. W. Tsui, C. R. Crawford, J. G. Ballard, and J. T. Hagius, "Estimation of geometrical parameters and collimator evaluation for cone beam tomography," Med. Phys., vol. 17, pp. 264-272, Mar.-Apr. 1990.

[3] J. Li, R. J. Jaszczak, H. Wang, K. L. Greer, and R. E. Coleman, "Determination of both mechanical and electronic shifts in cone beam SPECT," Phys. Med. Biol., vol. 38, pp. 743-743, 1993.

[4] T. Ma, R. Yao, Y. Shao, and R. Zhou, "A SVD-based method to assess the uniqueness and accuracy of SPECT geometrical calibration," IEEE Trans. Med. Imag., vol. 28, pp. 1929-1939, 2009.

[5] N. U. Schramm, G. Ebel, U. Engeland, T. Schurrat, M. Behe, and T. M. Behr, "High-resolution SPECT using multipinhole collimation," IEEE Trans. Nucl. Sci., vol. 50, pp. 315-320, 2003.

[6] L. von Smekal, M. Kachelriess, E. Stepina, and W. A. Kalender, "Geometric misalignment and calibration in cone-beam tomography," Med. Phys., vol. 31, pp. 3242-3266, 2004.

[7] H. Wang, M. F. Smith, C. D. Stone, and R. J. Jaszczak, "Astigmatic single photon emission computed tomography imaging with a displaced center of rotation," Med. Phys., vol. 25, pp. 1493-1501, 1998.

[8] S. T. Mahmood, K. Erlandsson, I. Cullum, and B. F. Hutton, "Design of a novel slit-slat collimator system for SPECT imaging of the human brain," Phys. Med. Biol., vol. 54, pp. 3433-3449, Jun. 7, 2009.

[9] S. D. Metzler and R. J. Jaszczak, "Simultaneous multi-head calibration for pinhole SPECT," IEEE Trans. Nucl. Sci., vol. 53, pp. 113-120, 2006.

[10] W. L. Rogers, N. H. Clinthorne, J. Stamos, K. F. Koral, R. Mayans, and J. W. Keyes et al., "SPRINT: A stationary detector single photon ring tomograph for brain imaging," IEEE Trans. Med. Imag., vol. 1, pp. 63-68, 1982.

[11] T. Ma, Y. Shao, M. Sajjad, H. Nabi, and R. Yao, "SPECT mouse imaging on a hybrid micro-PET/SPECT system," in Proc. Society of Nuclear Medicine Annu. Meeting, New Orleans, LA, 2008, vol. 49, p. 119.

[12] Y. Xia, T. Ma, X. Deng, Y. Liu, Y. Jin, and S. Murali et al., "A hybrid rotation-translation (HRT) scan scheme for in vivo animal SPECT imaging on a stationary scanner," in Proc. IEEE Nuclear Science Symp. Conf. Rec., Orlando, FL, 2009, pp. 2686-2689.

[13] S. D. Metzler, R. Accorsi, J. R. Novak, A. S. Ayan, and R. J. Jaszczak, "On-axis sensitivity and resolution of a slit-slat collimator," J. Nucl. Med., vol. 47, pp. 1884-1890, Nov. 1, 2006.

[14] R. Yao, T. Ma, and Y. Shao, "Derivation of system matrix from simulation data for an animal SPECT with slit-slat collimator," IEEE Trans. Nucl. Sci., vol. 56, pp. 2651-2658, 2009.

[15] R. Laforest, D. Longford, S. Siegel, D. F. Newport, and J. Yap, "Performance evaluation of the microPET-FOCUS-F120," IEEE Trans. Nucl. Sci., vol. 54, pp. 42-49, 2007.

[16] R. Accorsi, J. R. Novak, A. S. Ayan, and S. D. Metzler, "Derivation and validation of a sensitivity formula for slit-slat collimation," IEEE Trans. Med. Imag., vol. 27, pp. 709-722, May 2008.

[17] H. O. Anger, "Radioisotope cameras," in Instrumentation in Nuclear Medicine, J. G. Hine, Ed. New York: Academic, 1967, vol. 1, pp. $485-552$.

[18] E. C. Frey and B. M. W. Tsui, "Collimator-detector response compensation in SPECT," in Quantitative Analysis in Nuclear Medicine Imaging, H. Zaidi, Ed. New York: Springer, 2006, pp. 141-166.

[19] Y. Li, J. Oldendick, C. E. Ordonez, and W. Chang, "The geometric response function for convergent slit-slat collimators," Phys. Med. Biol., vol. 54, pp. 1469-1482, 2009.

[20] B. M. W. Tsui and G. T. Gullberg, "The geometric transfer function for cone and fan beam collimators," Phys. Med. Biol., vol. 35, pp. 81-93, 1990.

[21] S. D. Metzler, J. E. Bowsher, K. L. Greer, and R. J. Jaszczak, “Analytic determination of the pinhole collimator's point-spread function and RMS resolution with penetration," IEEE Trans. Med. Imag., vol. 21, pp. 878-887, Aug. 2002.

[22] G. Santin, D. Strul, D. Lazaro, L. Simon, M. Krieguer, and M. V. Martins et al., "GATE: A Geant4-based simulation platform for PET and SPECT integrating movement and time management," IEEE Trans. Nucl. Sci., vol. 50, pp. 1516-1521, 2003. 
[23] M. Bergeron, J. Cadorette, J. F. Beaudoin, M. D. Lepage, G. Robert, and V. Selivanov et al., "Performance evaluation of the LabPET APDbased digital PET scanner," IEEE Trans. Nucl. Sci., vol. 56, pp. 10-16, 2009.

[24] R. Yao, J. Seidel, C. A. Johnson, M. E. Daube-Witherspoon, M. V. Green, and R. E. Carson, "Performance characteristics of the 3-D OSEM algorithm in the reconstruction of small animal PET images," IEEE Trans. Med. Imag., vol. 19, pp. 798-804, 2000.

[25] F. J. Beekman, F. van der Have, B. Vastenhouw, A. J. A. van der Linden, P. P. van Rijk, and J. P. H. Burbach et al., "U-SPECT-I: A novel system for submillimeter-resolution tomography with radiolabeled molecules in mice," J. Nucl. Med., vol. 46, pp. 1194-1200, Jul. 2005.

[26] D. Beque, J. Nuyts, G. Bormans, P. Suetens, and P. Dupont, "Characterization of pinhole SPECT acquisition geometry," IEEE Trans. Med. Imag., vol. 22, pp. 599-612, 2003.
[27] M. Defrise, C. Vanhove, and J. Nuyts, "Perturbative refinement of the geometric calibration in pinhole SPECT," IEEE Trans. Med. Imag., vol. 27, pp. 204-214, 2008.

[28] S. D. Metzler, K. L. Greer, and R. J. Jaszczak, "Determination of mechanical and electronic shifts for pinhole SPECT using a single point source," IEEE Trans. Med. Imag., vol. 24, pp. 361-370, Mar. 2005.

[29] F. Noo, R. Clackdoyle, C. Mennessier, T. A. White, and T. J. Roney, "Analytic method based on identification of ellipse parameters for scanner calibration in cone-beam tomography," Phys. Med. Biol., vol. 45, pp. 3489-3508, Nov. 2000.

[30] P. Rizo, P. Grangeat, and R. Guillemaud, "Geometric calibration method for multiple-head cone-beam SPECT system," IEEE Trans. Nucl. Sci., vol. 41, pp. 2748-2757, 1994. 